\title{
Clinical Pharmacology
}

National Cancer Institute

\section{Source}

National Cancer Institute. Clinical Pharmacology. NCI Thesaurus. Code C16975.

The branch of pharmacology that studies the effectiveness and safety of drug treatment in humans. 